\title{
Critical review of ageing mechanisms and state of health estimation methods for battery performance
}

\author{
K. Saqli ${ }^{1}$, H. Bouchareb ${ }^{1}$, M. Oudghiri ${ }^{1}$, and N.K. M'Sirdi ${ }^{2}$ \\ 1 Sidi Mohammed Ben Abdellah University - National School of Applied Sciences, \\ Fez, Morocco, \\ saqli.khadijah@gmail.com, \\ 2 LIS - Informatics and Systems Laboratory (LIS CNRS 7020), Aix Marseille \\ University, CNRS, Kouider-Nacer.Msirdi@lis-lab.fr, \\ 13397 Marseille Cedex, France
}

\begin{abstract}
Battery Management System (BMS) is an essential component for lithium-ion battery-based devices. It provides a variety of functionalities that help improve the overall lifespan of the battery, including states estimation algorithms. An accurate state of health is one of the essential features an advanced BMS provides, in order to track long term performance and ensure reliable operation of the battery.

This paper aims to provide a structured review of ageing mechanisms that affect the battery performance and also some of the most relevant algorithms that were used in the recent literature to predict the actual health of the battery. Finally, and based on the different approaches that were studied evaluation criteria are proposed to help judge the performance of State Of Health (SOH) estimation algorithm.
\end{abstract}

Keywords: Battery management system - State Of Health · Lithiumion battery. Ageing mechanisms.

\section{Introduction}

To cope with climate changing and global warmth, communities have been promoting clean energy and they've been calling for a fossil-free society. According to the Environmental Protection Agency, $72 \%$ of carbon monoxide emissions come from automobiles only 1. Thus, interest in electric vehicles (EVs) has grown fast.

Electric vehicles are much cleaner and cheaper to drive 2. They usually use Lithium-ion batteries as an energy source, this type of chemistries presents the advantages of high energy density and low self-discharge compared with other rechargeable cells [4]. However, the capability to perform correctly and to store energy tends to decrease with time due to the ageing phenomenon. Therefore, Battery Management Systems (BMSs) are needed to boost the battery performance and improve its safety. 
In general, BMS is an electronic system that controls and monitors the battery for safe usage and long lifespan. It has three basic tasks: data acquisition, battery states estimation and electrical management of the measured input to take action when needed.

In addition to cell balancing, battery modelling is a significant contributor to BMS reliability and performance. An accurate battery model is required to predict the battery behaviour under different conditions and to provide a proper estimation of the battery states 3 .

Depending on the target application, the battery model can be reperesented using different approaches and can have different range of complexity. For electric vehicles, the battery model needs to be simple, computationally efficient and suitable for high discharge rates [4. Thus, two main categories of battery models were presented in the literature. The first category known as Reduced Order Model(ROM) and the second category is the Equivalent Circuit Model(ECM) 5].

Reduced-order models are simplified version of electrochemical battery model 6]. The governing PDE equations that describe the electrochemical reactions inside the battery are approximated into low order systems of ODEs equations using a set of Model Order Reduction(MOR) techniques [7]. The most basic electrochemical model is known as Single Particle Model(SPM) 40], it represents each electrode of the battery by a single spherical particle ignoring thermal effects, approximating spatial and time characteristics at the separator region to 0 and assuming all unknown states to be scalar and uniform 1. SPM are simple and they can be adopted for real time applications, however, they lack accuracy at high C-rate. To overcome this limitation an extended version of these model that incorporates the electrolyte dynamicshas been developed and they were proved to maintain a high accuracy prediction even at high C-rates conditions 8.

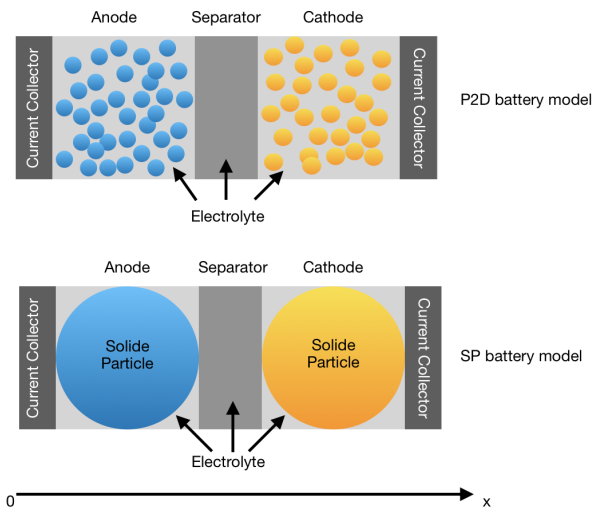

Fig. 1. P2D and Single Particle battery model 
Another version that aims to reduce the computational burden of the P2D model was presented in the literature as the Simplified P2D model (SP2D), it describes the dynamic concentration profiles derived from the P2D model to help improve the accuracy of the BMS 9 .

Equivalent circuit models on the other hand are widely used when developing a BMS for EV application thanks to their speed, simplicity and reasonable accuracy [10. Starting by the most basic model that represents the battery as an ideal voltage source mounted on series with internal resistance to describe the voltage polarization, researchers have include different electrical components, in the interest of building improved versions which take into account the dependence of the battery cell on state of charge, temperature, and other characteristics 11].

The most used ECM for estimating the battery states for EV applications uses: an ideal voltage source, series resistance and one or more RC networks to simulate the behaviour of the battery under load (see figure 2).

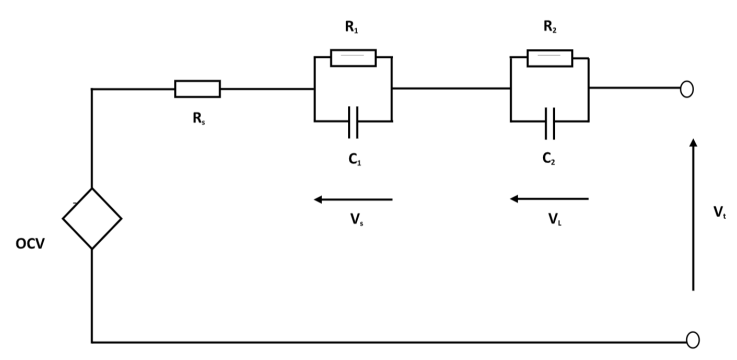

Fig. 2. Second order equivalent circuit model

Once the battery model has been decided, it can be used as an input for the states estimation algorithms whether it is the $\mathrm{SOC}, \mathrm{SOH}$ or any other state.

Battery State of Charge (SOC) is one of the essential parameters a BMS needs to determine. It represents an instant indication of the battery status that estimates the percentage of the available capacity. On the other hand, the State Of Health $(\mathrm{SOH})$, as its name implies, is a parameter that reflects the battery health and its ability to convey a specific performance compared with a fresh battery [13. Having control over ageing causes can help reduce the degradation effects, but once the $\mathrm{SOH}$ hits a certain percentage the battery can no longer be used for electric vehicles [55].

\subsection{SOH estimation in literature}

Numerous surveys have been conducted in recent years to classify the $\mathrm{SOH}$ estimation methods 13 17]. In order to have a clear vision of the different approaches that were developed or improved in the literature, M. Berecibar et al. 13] presented a detailed classification of battery $\mathrm{SOH}$ estimation methods, that 
arranges those approaches in two main categories depending on the way they were carried out. The strengths and weaknesses of each method were compared and analyzed, and the degradation mechanism was also presented. The authors of 14 review the estimation strategies and methodologies used in electric vehicles and classified them under three main categories that are: battery management related, vehicle energy management related and vehicle control related. Except for the battery state of charge(SOC), the rest of the estimation strategies are briefly discussed.

An extensive review of the methods for monitoring lithium-ion batteries in electric and hybrid vehicles is described in [15]. Based on an impressive 375 sources including technical and scientific literature, the estimation approaches for battery state of charge, state of health, capacity, impedance, available power and remaining useful life are provided.

Lucian Ungurean et al. 16 define a comprehensive, structured review of battery models and estimation methods used to determine the battery state of health. However, the ageing mechanisms of the battery are not presented. Same goes for Rui Xiong et al. [17 who provide a detailed presentation of battery SOH estimation algorithms with emphasis on their limits and strengths.

Before starting to define the state of health estimation approaches it's important to first outline the ageing mechanisms since, without having a clear insight of the ageing factors, we can not appropriately determine the battery health.

\section{Ageing mechanisms}

Degradation mechanisms affect battery health through reduction of total capacity and or expansion of equivalent series resistance, and observing those quantities that vary slightly and impact the battery performance as cell ages, is of great value to determine the battery state of health as accurately as possible.

Generally, unwanted side reaction and structural degradation that occurs inside the battery cell leads to capacity and power loss, and the complexity of the Li-ion based batteries makes the processes of their ageing at anodes and cathodes even more complicated [19].

Researchers have proved that ageing mechanisms at anodes differ from those at cathodes and their source can be either chemical or mechanical 20]. Therefore, the knowledge of these ageing processes is mandatory to control battery health as well as other parameters.

\subsection{Ageing in the negative electrode}

Depending on electrodes composition, safety properties and ageing ratio can differ from one cell to another. Therefore graphitic carbon materials are the most widely used as anode material, and thus, many research papers were devoted reviewing the ageing mechanisms of graphite-based anodes [21,22].

Ageing effects can occur during storage just after manufacturing and during use, and can be observed at three different scales that are: 
- Electrode/electrolyte interface :The main ageing factor that considerably affects the graphite electrode properties happens once the electrolyte comes into contact with the graphite, especially during the first charge inside of a battery manufacturing facility. The electrochemical stability window of the electrolyte does not include the voltages at which the graphite-based negative electrodes operate 24. Hence a protective layer is developed as a result of electrolyte decomposition. Known as Solid Electrolyte Interface (SEI) 23] .

Once it's formed, it encapsulates the graphite and blocks electrons from transport, to prevent any further electrolyte degradation [23]. The porous structure of this passivating layer allows the transition of Li-ion from the electrolyte to the particle, adding a resistance to the motion of Li-ion.

- Within the electrode particles : Ageing processes that occur inside the particles can have a minor negative impact on the electrode performance. The particles change volume when charging or discharging, due to the absorption and withdrawal of Li-ions 19 , leading to structural changes that can cause particles to crack and be less ordered compared to the original structure, which enables further SEI growth and active material loss 28 .

- Composite electrode : Volume changing affects the electrode porosity that facilitates the penetration into the bulk of the electrode. It is also considered as the main reason for the loss of contact between active cathode particles, that can be accelerated when over-discharging the battery cell, resulting in higher cell impedance and capacity loss 29 .

\subsection{Ageing in the positive electrode}

Aging can also occur at the positive electrode and can affect battery performance, and as for the negative electrode the chosen material impact significantly the battery performance. In terms of performance cost and abundance, most Lithium-ion battery cells use Lithium manganese oxides with spinel structure and lithium nickel cobalt mixed oxides with layered structures as the cathode material. An aged positive electrode material can have different effects that can be summarized as [30]:

- Film formation: The electrolyte oxidization and the decomposition of the electrode particles form a film layer called Solid Permeable Interface(SPI) that has different composition and properties from the anode SEI [31]. High temperatures induce the dissolution of metals from the electrode into the electrolyte accompanied by $\mathrm{CO}_{2}$ gas evolution breaking down the overall crystal structure of the electrode leading to capacity loss 19$]$.

- Loss of active material: The intercalation of lithium into the crystal structures of the cathode active materials causes mechanical stress that leads to strains of the metallic oxide particles and variation of their molar volume. These distortions are known as a phase transition. They are often reversible, and they proceed as lithium ions are inserted/extracted in/from the positive electrode. The most used lithium-ion battery cells have a structured 
layer, therefore when the battery is overcharged the lithium particles that hold these layers apart are extracted and the layers can fall on each other reducing lithium storage sites and causing capacity loss 36 .

- Metal dissolution : The dissolution phenomena of the metal from the electrode into the electrolyte results in capacity loss and can re-precipitate on the surface as a high-resistance film. The dissolved manganese particles moves to the anode and merge into the SEI interface enhancing the self-discharge of the battery cell. In general manganese dissolution can occur at low-potential, where trivalent manganese ions disproportionate into tetravalent and divalent ions, or at elevated potential known also as acid dissolution accelerated by hydrofluoric acid.

$$
2 \mathrm{Mn}(I I I) \longrightarrow \mathrm{Mn}(I V)+\mathrm{Mn}(I I)
$$

Battery cells age even when they are not used. Ageing can be separated into calendar ageing that represents degradation caused by battery storage, and cycle ageing that occurs when charging or discharging the battery.

\section{Health estimation}

The estimation of the battery state of health is usually associated with the determination of two essential indicators that are battery capacity and internal resistance, which respectively reflect the energy and power potential of the battery cell 34, 35. Different algorithms and processes can be applied to these parameters to track the degradation of the battery, and for better understanding of the performance of each approach, literature has proposed different classification for these methods.

\subsection{Internal resistance}

Internal resistance stems from the fact that a battery is not an ideal voltage source. It represents a direct indicator of the battery health that serves to spot any defects tending to damage the battery, and that explains the interest of researchers to measure this parameter.

Ageing causes the battery to lose its capacity and increase its internal resistance. Jinsong $\mathrm{Yu}$ et al [32], define the battery internal resistance based on changes in the voltage curve while applying a discharge current, and by using Ohm's law, the internal resistance can be approximated as follow:

$$
R=\frac{\Delta U}{\Delta I}
$$

Where $\Delta I$ represents the current step-change and $\Delta U$ is the corresponding voltage to the same step-change.

The internal resistance was proven to change with different factors such as battery size, chemistry, temperature and other parameters. Yingjie Chen et al 
33 considered that the battery internal resistance depends on the state of charge (SOC), the current rate and temperature, they conducted a constant current test under different temperatures to provide continuous R-SOC results.

\subsection{Impedance Spectroscopy method}

Battery impedance also is another parameter that we can use to determine the battery health, it comprises of impedance commitments from battery subcomponents(current collector, electrode and separator) and association between these sub-components. For example current collectors and separator can be viewed as complete resistive components, while impedance in electrodes comprised charge transfer, diffusion and contact resistance 36. Using electrochemical impedance spectroscopy, we can determine the actual value of this parameter, the reliability and of this method has motivated many researchers to estimate the battery states and also to predict the parameters of an electrochemical battery model 37 . However, since EIS is usually used with an equivalent circuit model to determine the battery state of health, the results are affected by the modification of the battery model due to ageing 38 .

\subsection{Coulomb counting}

Another way to estimate the battery $\mathrm{SOH}$ is by tracking the amount of charge that flows in or out during the charge-discharge process [16. It first calculates the capacity at discharge by integrating the value of discharge current in time, so a high precision current sensor is needed, then it uses this value to compute the $\mathrm{SOH}$ as follow:

$$
S O H[\%]=\frac{Q_{\max }}{Q_{\text {nominal }}} \times 100
$$

where $Q_{\max }$ is the maximum available capacity and $Q_{\text {nominal }}$ is the measured capacity. the accuracy of this approach requires high monitoring and storing capacity and a high-precision current sensor. Being not affected by the temperature, DOD or C-rate gained her wide popularity among methods that estimate the battery SOH and also SOC [13].

\subsection{Kalman filtering}

Kalman filtering is a sturdy algorithm that was used over history to estimate battery parameters and states. It's defined as an adaptive method that operates in two steps [13, first, the algorithm predict the current output variable, then, the estimation is updated to obtain more accurate results (3). 


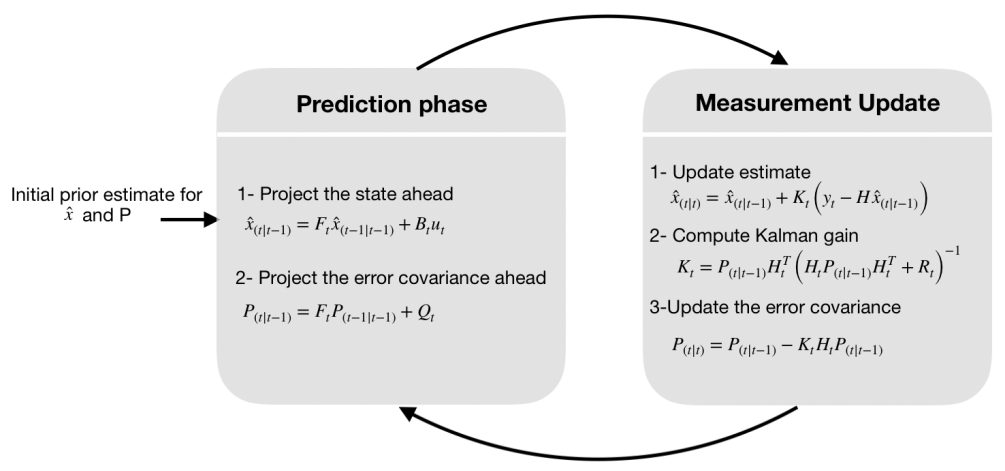

Fig. 3. Process of kalman filter

Where $\hat{x}$ is the estimated state, $\mathrm{F}$ is the state transition matrix, $\mathrm{B}$ is the control matrix, $\mathrm{u}$ is the input vector, $\mathrm{P}$ and $\mathrm{Q}$ are respectively the state and the process variance matrix, $\mathrm{y}$ is the output measurement, $\mathrm{K}$ is the Kalman gain, $\mathrm{H}$ is the measurement matrix and $\mathrm{R}$ is the measurement variance matrix.

Kalman filter can operate properly only when the system is linear, however, to deal with nonlinear systems extensions of this method have been developed such as extended Kalman filter (EKF), unscented Kalman filter (UKF), adaptive $\operatorname{EKF}(\mathrm{AEKF})$, dual extended kalman filter (DEKF) etc. 16].

Extended Kalman filter Extended Kalman filter is used when the transition and the measurement equations are not linear. A linearization process is applied at every time step to approximate the nonlinear system with a linear timevarying system (LTV) 46. The algorithm was widely used for battery modelling and states estimation 47, 48, thanks to its accuracy and speed.

In [49, the authors use a combination of extended Kalman filter and EIS algorithm to estimate the battery internal resistance and capacity to provide reliable information about its remaining stored energy.

50] perform a co-estimation of battery SOC and SOH using Lebesgue sampling based extended Kalman filter (LS-EKF) to reduce the computation cost when the states change slowly. The SOH and RUL were first predicted using LS-EKF. Then the obtained results are used to estimate the battery SOC.

Dual Extended Kalman filter As the name suggests, Dual extended KF (DEKF) is a combination of two extended Kalman filter that synchronously estimate the battery states and parameter 46] (see figure 4). N. Vassiliadis et al. propose a use-case life cycle analysis of DEKF for battery SOC and SOH prediction. The paper investigates the performance of the DEKF algorithm including different $\mathrm{SOH}$ stages and load dynamics. A comparison of the performance of DEKF versus a simple EKF shows a partial improvement in SOC accuracy. 


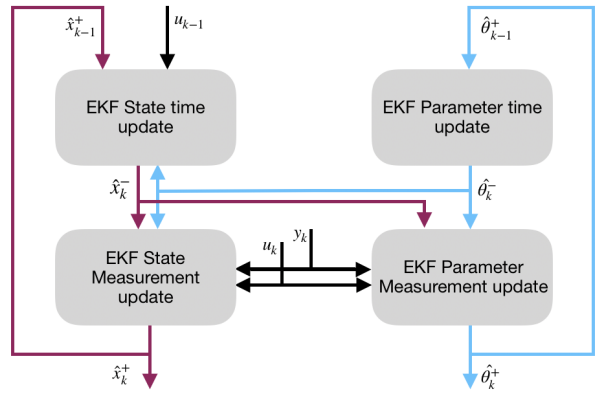

Fig. 4. DEKF Structure

The authors of [51] propose a combination of charging voltage curve and DEKF algorithm to estimate the battery SOC. The transformation of the charging voltage curve is used to determine the battery internal resistance and capacity, then DEKF algorithm is used to estimate the battery SOC. The results of this combination show an improvement in accuracy and elimination of the measurement noise.

Unscented Kalman filter Unscented Kalman filter also known as the sigma point Kalman filter is an algorithm where the state distribution is specified using a minimal set of carefully chosen sample points [52]. Unlike the EKF that loses its accuracy when dealing with highly nonlinear systems, the UKF uses the true nonlinear models to estimate the results as accurately as possible 53 .

54] used the UKF approach to predict the battery SOC, internal resistance and capacity, based on an improved Equivalent Circuit Model (ECM). The method was also used to evaluate different battery states(SOC, SOH, SOF), and was proved to be timesaving and efficient.

\subsection{Fuzzy Logic}

Fuzzy logic is a mathematical concept that models complex nonlinear systems and solves uncertainties in a given problem. The method uses crisp sets or fuzzy sets that allow elements to be in a set with a degree of membership. A membership function is used to define the relationship between a member of a set and its degree of membership that range from 0 to 1 39. Taking the battery state of health as an example, we can define the membership functions outputs as healthy, tolerable and not healthy.

In [40], the auther uses a fuzzy logic-controlled methodology to predict the battery $\mathrm{SOH}$, the process is divided into two parts, first the cell resistance and maximum capacity are determined based on voltage, current, temperature and time, Second using the resistance and the maximum capacity values $\mathrm{SOH}$ can be predicted by applying fuzzy logic. 
State of health estimation of Li-ion battery based on a fuzzy-logic system was also presented in 41], using temperature and the absolute value of the current the fuzzy logic system is constructed, which enhances the $\mathrm{SOH}$ value on both the calendar and cycling life.

\subsection{Support vector regression algorithm}

The support vector regression algorithm is a nonlinear generalization that implements a structural risk minimization principle to get a good generalization on a limited number of learning patterns 42 . It adopts the original machine learning algorithm to predict the output of a given input.

In [43], the authors used a novel support vector regression that takes the battery capacity as a state variable, and constant-current and constant voltage as an input variable of the system to predict the battery SOH.Furthermore, the model was combined with a particle filter to reduce the errors caused by measurement noises and to provide more accurate and robust result.

Duo yang et al 44 . proposed a novel SOH estimation approach. They started by building an improved battery model, which combines OCV modelling and Thevenin equivalent circuit model (ECM). The battery SOC was estimated using the joint extended Kalman filter-recursive-least squares algorithm. Then a reliable $\mathrm{SOH}$ estimation result was obtained using a combination of the support vector regression approach and the particle swarm optimization algorithm. The authors of [45] used Support Vector Machine (SVM)/Support Vector Regression (SVR) for classification/regression with less experimental data compared with the neural network. The SVM was used for battery classification and then they used SVR to estimate the remaining useful life of the battery.

\subsection{Least squares}

The least square algorithm is a widely used procedure to estimate the parameters of a given system. The basic problem is to identify the best fit function that minimizes the sum of errors between measured output and system response. To estimate the battery SOH, Gregory L.Pett [55] used an improved version of the RLS algorithm called the Weighted Total Least Squares estimation approach (WTLS). The author started by identifying the main drawback of using a standard least squares approach to estimate the battery health. Since this method doesn't take into consideration the uncertainty that we have on the input measurements (SOC) the author uses WTLS approach to determine the battery health with knowledge of both noises on the accumulate ampere-hour measurements and on the battery SOC. The method shows better results compared to a standard least squares algorithms and it's suitable for real-time implementation.

\subsection{Evaluation criteria}

The previous section has presented some of the recent commonly used approaches in the literature to estimate the actual health of the battery. The accuracy of 
the predicted $\mathrm{SOH}$ is a significant parameter that authors try to prove. However, this parameter only is insufficient to evaluate the estimation algorithm results. Thus we propose some criteria that can be used to judge the performance of a given algorithm:

- Battery model: When using an algorithm that predicts the battery states based on a specific battery model, the result precision can differ since some models lose theire accuracy with the use, due to different ageing mechanisms.

- Computation cost: it's an indication of whether the proposed approach is suitable for a real-time application, in another way if the method can be implemented in a BMS running an embedded system.

- Time cost: it defines the duration of the execution, the time needed to provide the result, which depends on the complexity of the algorithm and also the hardware specification.

- Estimation precision an indication of the calculation error, how much the result diverges from the actual one.

An extensive comparison of health estimation algorithms is presented in [13]. The author concludes that there is no unique or perfect method to estimate the battery health. The choice of method should be selected based on the target application, what's needed to be estimated and how much error tolerance is acceptable.

\section{Conclusion}

Lithium-ion batteries have been widely used as the main energy storage solution for EVs. To make the most of this technology, BMS was used to manage the battery performance and ensure the safe operation of the cells.

State of health estimation is a crucial parameter that helps batteries operate in a safe condition. This review presented the aging mechanisms that affect the Lithium batteries during their life to help build a basic understanding of the different factors that accelerate the process of aging and cause capacity fading. A description of the most relevant methods that were presented in the literature to estimate the battery internal resistance and capacity are provided, the review shows that there is no perfect health estimation algorithm and that the choice should be made based on the target application. Finally, the author proposes some evaluation criteria that can help judge the performance of the health prediction algorithm. 


\section{Acronyms}

AEKF
BMS
DEKF
ECM
EIS
EKF
EV
HEV
LS-EKF
LTV
OCV
P2D
ROM
RUL
SEI
SOC
SOF
SOH
SP2D
SPI
SPM
SVM
SVR
UKF
WTLS

Adaptive Extended Kalman Filter...................8 8

Battery management system .....................

Dual Extended Kalman Filter .....................

Equivalent Circuit Model .......................2

Equivalent Impedance Spectroscopy ............... 7

Extended Kalman Filter ......................... 8

Electric Vehicle .................................

Hybrid Electric Vehicle..........................6

Lebesgue sampling based Extended Kalman Filter....... $\overline{9}$

Linear Time-Varying ........................... 9

Open Circuit Voltage ...........................10

Pseudo two dimensional ..........................

Reduced Order Model..............................2

Remaining Useful Life.........................9

Solid Electrolyte Interface ....................... 5

State Of Charge ................................ 3

State Of Function ..............................

State Of Health ..................................

Simplified Pseudo two dimensional ................. 3

Solid Permeable Interface .......................

Single Particle Model ............................2

Support Vector Machine .........................10

Support Vector Regression .......................10

Unscented Kalman Filter........................

Weighted Total Least Square......................11

\section{References}

1. Vecotia Environment Protection Agency 2018 Retrieved from https://www.epa. vic.gov.au/your-environment/air/vehicle-emissions-and-air-quality

2. Kathryn Canepa, Scott Hardman, Gil Tal. An early look at plug-in electric vehicle adoption in disadvantaged communities in California. Transport Policy (2019).

3. Yinjiao Xing, Eden W. M. Ma, Kwok L. Tsui and Michael Pecht. Battery Management Systems in Electric and Hybrid Vehicles. Energies 2011, 4, 1840-1857.

4. Cheng Zhang, Kang Li, Sean Mcloone, Zhile Yang. Battery Modelling Methods for Electric Vehicles - A Review. 2014 European Control Conference (ECC).

5. Abbas Fotouhi, Daniel J. Auger, Karsten Propp, Stefano Longo, Mark Wild. A review on electric vehicle battery modelling: From Lithium-ion toward Lithium-Sulphur. Renewable and Sustainable Energy Reviews 56 (2016) 1008-1021.

6. Jokar, A., Rajabloo, B., Désilets, M. and Lacroix, M. Review of simplified Pseudo-two-Dimensional models of lithium-ion batteries. Journal of Power Sources, $327,(2016) 44-55$. 
7. Guodong Fan, Xiaoyu Li, and Marcello Canova. A Reduced-Order Electrochemical Model of Li-Ion Batteries for Control and Estimation Applications

8. Scott J. Moura, Federico Bribiesca Argomedo, Reinhardt Klein,Anahita Mirtabatabaei, and Miroslav Krstic. Battery State Estimation for a Single Particle Model With Electrolyte Dynamics. IEEE Transactions on Control Systems Technology, 25(2), 453-468.

9. Fan, G., Li, X., Canova, M. A Reduced-Order Electrochemical Model of Li-Ion Batteries for Control and Estimation Applications. IEEE Transactions on Vehicular Technology, 67(1), 76-91. 2018.

10. M'Sirdi N.K., Belhani A., Naamane A. (2012) Battery Models for Estimation of State of Charge by Sliding Mode Observer. Sustainability in Energy and Buildings. Smart Innovation, Systems and Technologies, vol 12. Springer, Berlin, Heidelberg.

11. Kemper, P., Li, S. E., Kum, D. Simplification of pseudo two dimensional battery model using dynamic profile of lithium concentration. Journal of Power Sources, 286, 510-525. 2015

12. Min Chen and G. Rincon-Mora. Accurate Electrical Battery Model Capable of Predicting Runtime and I-V Performance. IEEE Trans on Energy Conversion, Vol. 21, No. 2, June 2006.

13. M. Berecibar, I. Gandiaga, I. Villarreal, N. Omar, J. Van Mierlo, P. Van den Bossche. Critical review of state of health estimation methods of Li-ion batteries for real applications. Renewable and Sustainable Energy Reviews 56 (2016) 572-587

14. Cuma, M. U., Koroglu, T. A comprehensive review on estimation strategies used in hybrid and battery electric vehicles. Renewable and Sustainable Energy Reviews, (2015), 42, 517-531.

15. Wladislaw Waag, Christian Fleischer, Dirk Uwe Sauer. Critical review of the methods for monitoring of lithium-ion batteries in electric and hybrid vehicles. Journal of Power Sources 258 (2014) 321-339

16. Lucian Ungurean, Gabriel Cârstoiu, Mihai V. Micea and Voicu Groza. INTERNATIONAL JOURNAL OF ENERGY RESEARCH (2016)

17. Rui Xiong, Linlin Li, Jinpeng Tian. Towards a smarter battery management system: A critical review on battery state of health monitoring methods. Journal of Power Sources 405 (2018) 18-29.

18. Andrew W. Thompson Review of Lithium Ion Battery Degradation Mechanisms, Models, and their Economic Implications. December 2013.

19. J. Vetter, P. Novak, M.R. Wagner, C. Veit, K.-C. Moller, J.O. Besenhard, M. Winter b, M. Wohlfahrt-Mehrens, C. Vogler, A. Hammouche. Ageing mechanisms in lithium-ion batteries. Journal of Power Sources 147 (2005) 269-281

20. Anthony Barré, Benjamin Deguilhem, Sébastien Grolleau, Mathias Gérard, Frédéric Suard, Delphine Riu. A review on lithium-ion battery ageing mechanisms and estimations for automotive applications. Journal of Power Sources 241 (2013) 680-689

21. Y.P Wu, E. Rahm, R.Holze. Carbon anode materials for lithium ion batteries. Journal of Power Sources 114(2003) 228-236

22. Yemeserach Mekonnen, Aditya Sundararajan, Arif I. Sarwat. A Review of Cathode and Anode Materials for Lithium-Ion Batteries. 978-1-5090-2246-5/16/ 2016 IEEE

23. Pallavi Verma, Pascal Maire, Petr Novák. A review of the features and analyses of the solid electrolyte interphase in Li-ion batteries. Electrochimica Acta 55 (2010) $6332-6341$

24. Shengshui Zhang, Michael S. Ding, Kang Xu, Jan Allen, and T. Richard Jow. Understanding Solid Electrolyte Interface Film Formation on Graphite Electrodes. Electrochemical and Solid-State Letters, 4 (12) A206-A208 2001. 
25. M. Koltypin, D. Aurbach, L. Nazar b, B. Ellis. More on the performance of $\mathrm{LiFePO} 4$ electrodes - The effect of synthesis route, solution composition, aging, and temperature. Journal of Power Sources 174 (2007) 1241-1250.

26. S.S. Zhang, K. Xu, T.R. Jow. The low temperature performance of Li-ion batteries. Journal of Power Sources 115 (2003) 137-140.

27. Ravi N. Methekar, Paul W. C. Northrop, Kejia Chen, Richard D. Braatz, and Venkat R. Subramanian. Kinetic Monte Carlo Simulation of Surface Heterogeneity in Graphite Anodes for Lithium-Ion Batteries: Passive Layer Formation. Journal of The Electrochemical Society, 158 (4) A363-A370 (2011).

28. J. Vetter, P. Novák, M. Wagner, C. Veit, K.-C. Möller, J. Besenhard, M. Winter, M. Wohlfahrt-Mehrens, C. Vogler, and A. Hammouche. Ageing mechanisms in lithiumion batteries. Journal of Power Sources, vol. 147, no. 1-2, pp. 269- 281, 2005.

29. Yifeng Wang, Xiaodong Guo, Steve Greenbaum, Jun Liu and Khalil Amine. Solid Electrolyte Interphase Formation on Lithium-Ion Electrodes. Electrochem. SolidState Lett. 2001, Volume 4, Issue 6, Pages A68-A70.

30. M. Dubarry, C. Truchot, B. Y. Liaw, K. Gering, S. Sazhin, D. Jamison, and C. Michelbacher. Evaluation of commercial lithium-ion cells based on composite positive electrode for plug-in hybrid electric vehicle applications. Part II. Degradation mechanism under 2 C cycle aging. Journal of Power Sources, vol. 196, pp. 10 336-10 343, 2011.

31. S. Malmgren, K. Ciosek, M. Hahlin, T. Gustafsson, M. Gorgoi, H. Rensmo, and K. Edström. Comparing anode and cathode electrode/electrolyte interface composition and morphology using soft and hard X-ray photoelectron spectroscopy. Electrochimica Acta, vol. 97, pp. 23 - 32, 2013.

32. Jinsong Yu, Baohua Mo, Diyin Tang, Jie Yang, Jiuqing Wan and Jingjing Liu. Indirect State-of-Health Estimation for Lithium-Ion Batteries under Randomized Use. Energies, 2017.

33. Yingjie Chen, Xu Liu, Geng Yang, Hua Geng. An Internal Resistance Estimation Method of Lithium-ion Batteries with Constant Current Tests Considering Thermal Effect. IECON 2017-43rd Annual Conference of the IEEE Industrial Electronics Society.

34. Wladislaw Waag, Stefan Käbitz, Dirk Uwe Sauer. Experimental investigation of the lithium-ion battery impedance characteristic at various conditions and aging states and its influence on the application. Applied Energy 102 (2013) 885-897.

35. Gregory L. Plett. Dual and Joint EKF for Simultaneous SOC and SOH Estimation. 2004

36. Evelina Wikner. THESIS FOR THE DEGREE OF LICENTIATE OF ENGINEERING. Lithium ion Battery Aging: Battery Lifetime Testing and Physics-based Modeling for Electric Vehicle Applications

37. Shengbo Eben Li, Baojin Wang, Huei Peng, Xiaosong Hu. An electrochemistrybased impedance model for lithium-ion batteries. Journal of Power Sources 258 (2014) 9-18

38. Hassan Shabbir, William Dunford, Tina Shoa. State of Health Estimation of Li-Ion batteries using Electrochemical Impedance Spectroscopy. 2017 IEEE

39. Patil Pallavi D, Prof Patel J. A Comprehensive Review On Fuzzy Logic System. International Journal Of Engineering And Computer Science ISSN:2319-7242 Volume 3 Issue 11 November, 2014 Page No. 9160-9165

40. Jonghoon Kim, Fuzzy Logic-Controlled Online State-of-Health (SOH) Prediction in Large Format LiMn2O4 Cell for Energy Storage System (ESS) Applications. 2014 IEEE International Conference on Industrial Technology (ICIT), Feb. 26 - Mar. 1, 2014, Busan, Korea 
41. Ali ZENATI,Philippe DESPREZ, Hubert RAZIK, Stéphane RAEL. A Methodology to Assess the State Of Health of Lithium-ion Batteries Based on the Battery's Parameters and a Fuzzy Logic System. 2012 IEEE International Electric Vehicle Conference

42. Debasish Basak, Srimanta Pal and Dipak Chandra Patranabis. Support Vector Regression. Neural Information Processing - Letters and Reviews Vol. 11, No. 10, October 2007.

43. Jingwen Wei, Guangzhong Dong and Zonghai Chen. Remaining Useful Life Prediction and State of Health Diagnosis for Lithium-ion Batteries Using Particle filter and Support Vector Regression. IEEE Transactions on Industrial Electronics, 65(7), 5634-5643. 2018.

44. Duo Yanga, Yujie Wanga, Rui Pana, Ruiyang Chenb, Zonghai Chen. State-ofhealth estimation for the lithium-ion battery based on support vector regression. Applied Energy (2017).

45. Meru A. Patil, Piyush Tagade, Krishnan S. Hariharan, Subramanya M. Kolake, Taewon Song, Taejung Yeo, Seokgwang Doo. A novel multistage Support Vector Machine based approach for Li ion battery remaining useful life estimation. Applied Energy 159 (2015) 285-297

46. Gregory L. Plett. Extended Kalman filtering for battery management systems of LiPB-based HEV battery packs Part 1. Background. Journal of Power Sources 134 (2004) 252-261.

47. Afshar, S., Morris, K. and Khajepour, A. State of Charge estimation via extended Kalman filter designed for electrochemical equations. IFAC-PapersOnLine, 50(1), 2152-2157. (2017).

48. Ivan Jokić, Student Member, Žarko Zečević and Božo Krstajić. State-of-Charge Estimation of Lithium-ion Batteries using Extended Kalman filter and Unscented Kalman filter. 23rd International Scientific-Professional Conference on Information Technology. 2018.

49. M. Urbain, S. Raël, B. Davat, P. Desprez. State Estimation of a Lithium-Ion Battery Through Kalman Filter. IEEE Power Electronics Specialists Conference. 2007.

50. Wuzhao Yan, Bin Zhang, Guangquang Zhao, Shijie Tang, Guangxing Niu, and Xiaofeng Wang. Battery Management System with Lebesgue Sampling-Based Extended Kalman Filter. IEEE Transactions on Industrial Electronics ( Volume: 66 , Issue: 4 , April 2019 )

51. Limei Wang, Dong Lu, Qiang Liu, Liang Liu, Xiuliang Zhao. State of charge estimation for LiFePO4 battery via dual extended kalman filter and charging voltage curve. Electrochimica Acta 296 1009-1017. 2019.

52. Manojkumar Rampelli, Debashisha Jena. Advantages of unscented Kalman filter over extended kalman filter in dynamic state estimation of power system network. Michael Faraday IET International Summit: MFIIS-2015, September 12 - 13, 2015, Kolkata, India.

53. Amin Sedighfar, M. R. Moniri. Comparison of Three Well-known Filters for the Battery State of Healt Estimation Application. 4th International Conference on Frontiers of Signal Processing. 2018.

54. Fei Zhang, Guangjun Liu, Senior and Lijin Fang. Battery State Estimation Using Unscented Kalman Filter. IEEE International Conference on Robotics and Automation Kobe International Conference Center. 2009.

55. Plett, G. L. Recursive approximate weighted total least squares estimation of battery cell total capacity. Journal of Power Sources, 196(4), 2319-2331. 2011. 